\title{
Geografía del acceso desigual a los servicios de energía en los hogares de México ${ }^{\text {th }}$
}

\section{The Geography of unequal access to energy services in Mexican households}

\author{
Rigoberto García Ochoa*a $^{*}$, Daniel Itzamna, Avila-Ortega ${ }^{3}$, Jordi Cravioto \\ ${ }^{a}$ El Colegio de la Frontera Norte, Calle Reforma No.528, Col. Del Rosario, CP 84020, Nogales, Sonora, México \\ ${ }^{b}$ Centro Mexicano de Ecología Industrial, CP 61503, Zitácuaro, Michoacán, México \\ ${ }^{c}$ Institute of Advanced Energy, Kyoto University, Gokasho, Uji, Kioto 611-0011, Japón
}

\section{Resumen}

Partiendo de la Meta 7.1 del objetivo siete de la Agenda 2030, la cual consiste en garantizar el acceso universal a los servicios de energía, se plantea como hipótesis que el acceso universal a la electricidad, como lo establece el indicador 7.1.1, es una condición necesaria pero insuficiente para reflejar el acceso real a los servicios de energía. El objetivo de este trabajo es analizar la distribución espacial de los niveles de acceso a diferentes servicios de energía en los hogares de México. Utilizando datos a nivel municipal con la Encuesta Intercensal 2015, se identificaron seis categorías y diez dominios asociados a los servicios de energía. Se analizaron en cada una los niveles de acceso aplicando técnicas estadísticas. Los resultados muestran una clara desigualdad espacial-territorial en el acceso, así como una notoria diferencia entre el nivel de acceso a la electricidad y a los servicios de energía. Se propone que el conocimiento generado en este trabajo pueda convertirse en un insumo para un estudio más sistemático de la relación entre servicios de energía y otras dimensiones socio-económicas y ambientales del consumo de energía, sobre todo para concebir el desarrollo humano sostenible más allá del acceso universal a la electricidad.

Palabras clave: ODS; energía; servicios; acceso; México; geografía

\begin{abstract}
The Sustainable Development Goal 7 states through target 7.1 that by 2030 we should 'ensure universal access to affordable, reliable and modern energy services'. We hypothesize that Indicator 7.1.1 centred on universal electricity provision is certainly a necessity, yet it alone is insufficient to truly reflect the access to energy services. The objective of this study is to present a spatial analysis of the degree of access to different energy services. Using data from Mexican households, we define six categories of energy services and ten domains to measure the access levels, and analyse their distribution at the national scale. We find a clear geography of unequal access to energy services in Mexican households. In addition, a sharp difference between access to electricity and energy services supports our hypothesis. We conclude discussing how our proposal might set the basis for further studies on energy services in connection with other socio-economic and environmental dimensions. We suggest conceiving sustainable development beyond only guaranteeing universal access to electricity.
\end{abstract}

Keywords: SDG; energy; energy services; access; Mexico; geography

\section{Introducción}

El estudio del acceso a los servicios de energía es un tema que está cobrando cada vez más relevancia científica, tanto en

(C) R. García Ochoa, D. I. Avila-Ortega, J. Cravioto This is an Open Access article distributed under the terms of the Creative Commons Attribution License (https://creativecommons.org/licenses/by-nc-sa/4.0/), which permits non-commencial sharing of the work and adaptions, provided the original work is properly cited and the new creations are licensed under identical terms.

*E-mail address: rigo@colef.mx
México como en el mundo, debido a las implicaciones que tiene el uso de energía para el desarrollo sostenible (Kalt et al., 2019; Fell, 2017; Cravioto et al., 2014). Como tema de política pública, este vínculo se manifiesta explícitamente en la Agenda 2030 de desarrollo sostenible, la cual contempla en su objetivo siete: "garantizar el acceso a energía asequible, confiable, sostenible y moderna para todos" (NU, 2018), tema que cobra aún mayor relevancia en la actualidad por la pandemia del COVID19 , ya que los servicios energéticos son indispensables para la habitabilidad de los hogares en un entorno de confinamiento y 
distanciamiento social.

Un tema que ha sido poco estudiado en México, relacionado con el acceso a los servicios de energía, es su dimensión espacial y territorial, lo cual representa, desde nuestra perspectiva, un problema de investigación relevante que se sustenta en dos ejes principales. El primero es que la heterogeneidad económica, social y climática del país nos permite inferir que existe una diferenciación territorial en el acceso y uso de los servicios de energía. Esto puede ser debido a la capacidad económica en los hogares para cubrir los gastos en energía y los servicios que proporciona. También puede deberse a las diversas zonas climáticas que existen en el país (García, 2004), un factor que determina la intensidad de uso del servicio de climatización entre otros. El segundo tiene que ver con la diferenciación que existe entre acceso a la energía y acceso a los servicios que brinda la energía, en el sentido que, si bien México es un país donde más del $99 \%$ de la población cuenta con acceso a la electricidad, hay un proporción significativa de hogares que no pueden usar los diferentes servicios que brinda, como el calentamiento de agua, el acceso a la información y el entretenimiento, el confort térmico, y la iluminación (García y Bracamonte, 2019). Este escenario requiere un tratamiento espacial-territorial para conocer dónde y cómo se manifiestan estas diferencias.

Ante esta realidad, el objetivo de este trabajo es analizar la distribución espacial, a nivel municipal, de los niveles de acceso a los diferentes servicios de energía en los hogares mexicanos. El conocimiento generado con este trabajo puede convertirse, desde nuestra perspectiva, en un insumo empírico para analizar la relación entre energía y desarrollo sustentable en México.

\section{Metodología}

La estrategia metodológica consistió en tres etapas que explicamos a continuación.

\subsection{Fuentes de información}

El primer paso fue identificar la principal fuente de información para este estudio: La Encuesta Intercensal 2015 del Instituto Nacional de Estadística y Geografía (INEGI, 2016). El tamaño de muestra de este instrumento es de 6.1 millones de viviendas, con lo cual los resultados obtenidos son representativos a nivel nacional, entidad federativa, municipio y para cada una de las localidades con 50 mil o más habitantes. Esto garantiza la representatividad estadística de nuestros resultados, los cuales son presentados a nivel municipal.

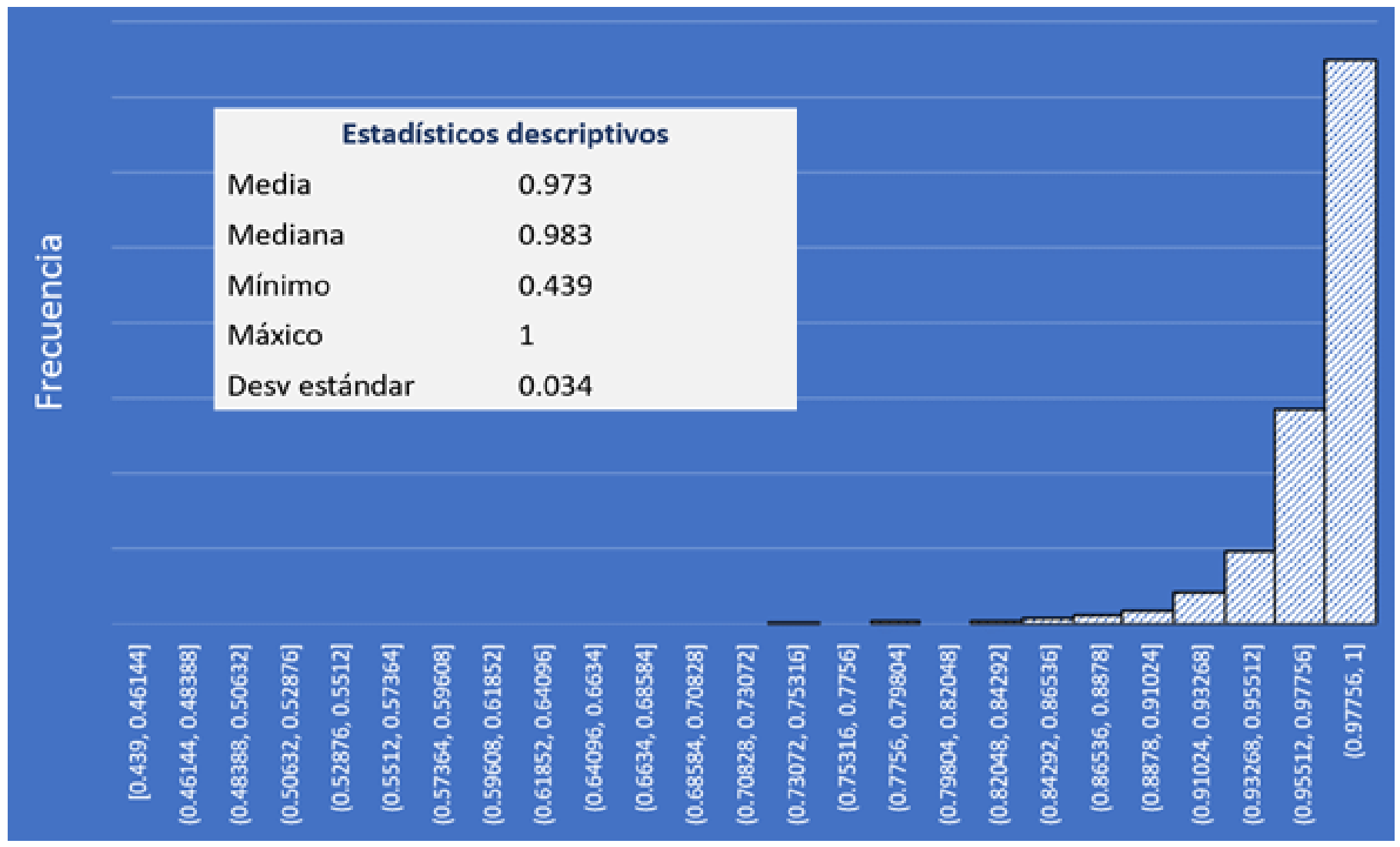

Figura 1: Estadísticos descriptivos e histograma del acceso a la electricidad a nivel municipal en México. Fuente: elaboración propia con información de INEGI (2016) / Figure 1. Descriptive statistics and histogram of electricity access level at the municipal level in Mexico. Source: own elaboration with information from INEGI (2016) 


\section{2. $\quad$ Niveles de acceso a la electricidad}

Enseguida procedimos a clasificar a los municipios de México en función de sus niveles de acceso a la electricidad. Para ello, exploramos primero los estadísticos descriptivos, encontrando los resultados que se muestran en la Figura 1. Destacamos en primer lugar que la mediana $(98.3 \%)$ es mayor a la media $(97.3 \%)$, de ahí que el histograma muestre una distribución sesgada a la izquierda, lo cual indica que la mediana es un mejor indicador de la tendencia central para la distribución del acceso a la electricidad por municipio. En segundo lugar, vemos que el rango es relativamente amplio $(56.1 \%)$, aunque la frecuencia de casos a la izquierda de la mediana es un claro indicativo de que son pocos los municipios con niveles muy bajos de acceso a la electricidad.

Con base en esta breve exploración descriptiva, procedimos clasificar a los municipios en función de sus niveles de acceso a la electricidad. Para ello, aplicamos el método de análisis de conglomerados $\mathrm{k}$ medias, técnica de análisis multivariante que agrupa los objetos (municipios en este caso) en conglomerados con base en dos características fundamentales (Hair et al., 2007). La primera es que se maximiza la homogeneidad interna de cada uno, lo cual significa que los municipios que conformen cada conglomerado tendrán niveles de acceso muy similares. La segunda es que se maximiza la heterogeneidad intergrupal, es decir, los niveles de acceso a la electricidad entre los municipios de cada conglomerado serán significativamente diferentes.

Tomando en cuenta que este método no ofrece el número adecuado de conglomerados, aplicamos primero el método de análisis de conglomerados jerárquico, ya que la literatura especializada recomienda utilizar este método en la muestra del total de casos analizados para conocer la cantidad óptima de grupos o conglomerados de municipios (Hair et al., 2007). Repetimos este procedimiento varias veces, tomando muestras diferentes que variaron entre 10 y $40 \%$ del total de casos, y encontramos de manera consistente que se formaban cinco conglomerados. Con este resultado, aplicamos después el método de análisis $\mathrm{k}$ medias al total de los casos y asignamos a los cinco grupos o conglomerados de municipios la siguiente clasificación, en función del nivel de electrificación: i) Muy alto, ii) Alto, iii) Medio, iv) Bajo y iv) Muy bajo.

\subsection{Construcción de indicadores de acceso a los servicios de energía}

El siguiente paso consiste en construir los indicadores de acceso a los servicios de energía en los hogares. Basándonos

\begin{tabular}{|c|c|c|}
\hline Servicio de energía & Dominio & Umbral de satisfacción \\
\hline Electricidad & Acceso a energía & Vivienda con conexión a electricidad \\
\hline Iluminación & Luminarias eficientes & $\begin{array}{l}\text { Tener el } 90 \% \text { o más luminarias ahorradoras del total en } \\
\text { el hogar }\end{array}$ \\
\hline \multirow{2}{*}{$\begin{array}{l}\text { Información, comunicación, } \\
\text { y entretenimiento }\end{array}$} & Televisión & Contar con al menos un televisor \\
\hline & Internet & $\begin{array}{l}\text { Contar con al menos una computadora con acceso a } \\
\text { internet }\end{array}$ \\
\hline \multirow[t]{2}{*}{ Alimentación } & Cocción de alimentos & $\begin{array}{c}\text { Contar con estufa de gas o eléctrica, o estufa de leña con } \\
\text { chimenea }\end{array}$ \\
\hline & Refrigeración de alimentos & Contar con un refrigerador \\
\hline \multirow[t]{2}{*}{ Higiene y limpieza } & Agua caliente & Contar con un calentador de agua \\
\hline & Lavadora de ropa & Contar con una lavadora \\
\hline \multirow[t]{2}{*}{ Confort térmico } & Vivienda adecuada & $\begin{array}{c}\text { Contar con lo siguiente en la vivienda: } 1 \text {. Paredes } \\
\text { construidas a base de block, tabique de ladrillo, piedra, } \\
\text { cantera, cemento o concreto, madera o adobe. } 2 \text {. Techos } \\
\text { construidos a base de losa de concreto, vigueta con } \\
\text { bovedilla, teja, o terrado con viguería.3. Piso construido } \\
\text { a base de cemento o firme, madera, mosaico u otro } \\
\text { recubrimiento }\end{array}$ \\
\hline & Aire acondicionado & $\begin{array}{l}\text { Contar con al menos un equipo de aire acondicionado } \\
\text { (portátil, ventana, central, aire evaporativo, "mini-split", } \\
\text { "inverter") }\end{array}$ \\
\hline
\end{tabular}

*Fuente: Elaboración propia. 
en las propuestas de García y Bracamonte (2019) y Cravioto (2020), definimos a los servicios de energía como aquellos beneficios que produce el uso de energía para el bienestar humano en los hogares. De acuerdo con la información recabada de INEGI (2016), los indicadores correspondientes a los servicios de energía que podemos identificar en México, así como sus niveles de satisfacción (umbrales), son los que se muestran en la Tabla 1, que corresponden a cinco servicios y nueve dominios. El análisis de estos indicadores nos permitirá probar la hipótesis de trabajo, es decir, la existencia de una desigualdad territorial en el acceso a los diferentes servicios que brinda en los hogares, independientemente de que tengan acceso a la electricidad. Si probamos esta hipótesis descriptiva, los resultados podrán servir como un insumo empírico para pensar que la Meta 7.1, del objetivo siete, de la Agenda 2030 debería ir más allá del acceso universal a la electricidad. Esto es válido, desde luego, si el objetivo es mejorar la calidad de vida y bienestar de la población.

\section{Resultados}

La Tabla 2 resume la información de los cinco grupos de municipios identificados cuya localización geográfica se muestra en la Figura 2. Vemos que 1,857 municipios, aproximadamente $76 \%$ del total nacional, tienen un nivel muy alto de acceso a la electricidad; mientras que 488 municipios, $20 \%$ del total, tienen un nivel alto. Estos resultados muestran algo conocido: que la gran mayoría de los municipios de México tienen niveles altos o muy altos de electrificación, acercándose incluso al acceso universal. Solo 84 municipios tienen un nivel medio, pocos un nivel bajo (14), y muy pocos, sólo tres, un nivel muy bajo. A primera vista, podría pensarse que es relativamente sencillo alcanzar la meta 7.1 de acceso universal a la electricidad y, con ello, el objetivo siete de la Agenda 2030 que busca "garantizar el acceso a una energía asequible, segura, sostenible y moderna para todos". Sin embargo, el escenario cambia significativamente cuando nos enfocamos en el acceso a los servicios de energía (Figura 3). Si bien es necesario alcanzar la electrificación universal, es insuficiente ya que la energía permite el acceso a una serie de servicios necesarios para mejorar la calidad de vida y bienestar de la población, servicios que van más allá del mero acceso a la electricidad. Es decir, el sólo acceso a la electricidad no garantiza los diferentes servicios que nos puede brindar.

En este sentido, si observamos los 1,857 municipios de México que presentan los niveles más altos de electrificación (Figura $2 \sqrt{1}$ encontramos que $98.6 \%$ de las viviendas de estos municipios cuentan con electricidad, pero el acceso general a los servicios de energía es considerablemente menor (Figura 3). Por dominio, se observa que casi el $95 \%$ del total de viviendas cuentan con televisión, pero en el resto de los dominios de los 5 servicios de energía se observan valores menores. Otros dominios con niveles de acceso alto fueron la cocción (estufa) y refrigeración de alimentos (refrigerador), la vivienda adecuada y lavadora de ropa. Con un nivel intermedio se encuentran el agua caliente (calentador de agua) y luminarias eficientes. Finalmente, el internet y el aire acondicionado son dominios con nivel de acceso más bajo. De manera similar, en los 488 municipios con nivel de electrificación alto, donde aproximadamente $95 \%$ del total de hogares cuentan con energía eléctrica, comprobamos también que una proporción importante de estos hogares no cuentan con acceso a ciertos servicios de energía. En este caso, identificamos un patrón similar al del grupo anterior, con la diferencia que los niveles de acceso en todos los dominios son significativamente menores. Por último, se encuentran los grupos de municipios con los niveles de acceso a electricidad medio, bajo, y muy bajo, y observamos la misma tendencia. Sin embargo, los niveles de acceso son aún menores a los dos grupos anteriores, llegando incluso a niveles extremadamente bajos, sobre todo en el acceso a internet, aire acondicionado, agua caliente y una vivienda adecuada.

En suma, podemos decir que, de los 5 servicios de energía analizados, 'alimentación' (cocción y refrigeración de alimentos) es en el que se observó mayor nivel de acceso, considerando los 5 niveles de electrificación analizados. 'Información, comunicación y entretenimiento' el segundo con mayor nivel de acceso, en mayor medida por el acceso al televisor. En contraparte, el acceso a internet fue consistentemente muy bajo,

\footnotetext{
${ }^{1}$ Véase el mapa interactivo de este artículo, en el cual se muestra la información de los servicios de energía correspondientes a cada uno de los municipios de acuerdo con su nivel de acceso a la electricidad.
}

\begin{tabular}{|c|c|c|c|c|c|}
\hline \multirow[t]{2}{*}{ Nivel de acceso } & \multicolumn{2}{|c|}{ Municipios } & \multicolumn{3}{|c|}{$\%$ viviendas con acceso } \\
\hline & Cantidad & $\%$ & Mínimo & Máximo & Media \\
\hline Muy alto & 1,857 & 75.9 & 96.6 & 100.0 & 98.6 \\
\hline Alto & 488 & 20.0 & 91.1 & 96.6 & 94.9 \\
\hline Medio & 84 & 3.4 & 81.5 & 90.9 & 87.8 \\
\hline Bajo & 14 & 0.6 & 66.1 & 79.7 & 75.3 \\
\hline Muy bajo & 3 & 0.1 & 43.9 & 60.8 & 52.2 \\
\hline Total & 2,446 & 100.0 & & & \\
\hline
\end{tabular}

*Fuente: elaboración propia

Tabla 2: Niveles de acceso a la electricidad y distribución municipal en México / Table 2: Electricity access level and municipality distribution in Mexico 


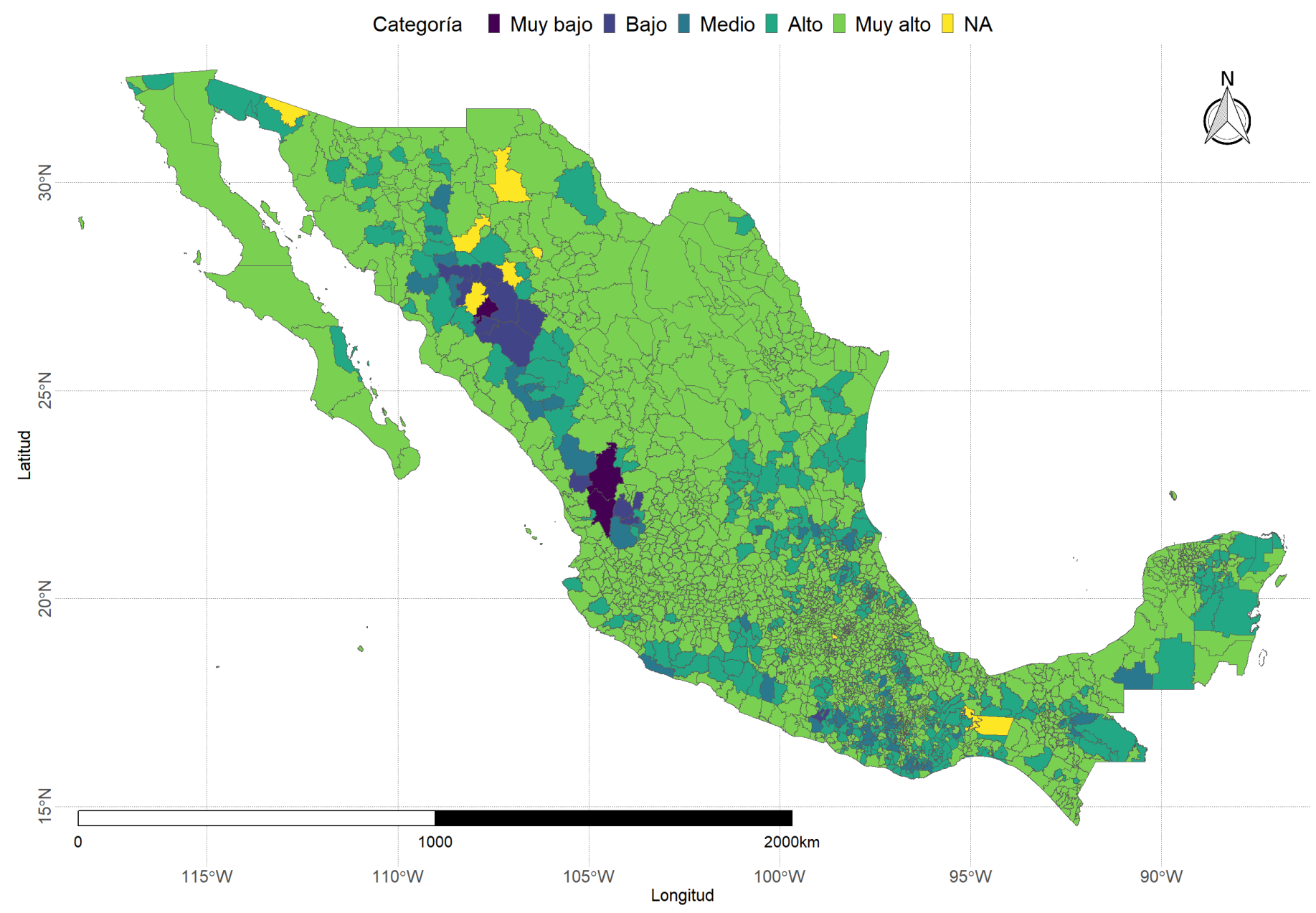

Figura 2: Distribución espacial de los municipios de México por nivel de acceso a electricidad en las viviendas. Fuente: elaboración propia con información de INEGI (2016)/ Figure 2: Mexican municipalities spatial distribution by electricity acccess in households. Source: Own elaboration with data from INEGI (2016)

inclusive en aquellos municipios con alto nivel de electrificación. Otro resultado similar se observó en el 'confort térmico' aunque con cambios más radicales en los niveles de vivienda adecuada pero un acceso a equipos de aire acondicionado en general muy bajo, aunque a diferencia del internet, se entiende que la existencia de un equipo de aire acondicionado está más sujeta a condiciones geográficas y climáticas. En 'higiene y limpieza' también se observó un cambio drástico en los niveles de acceso de estar ligeramente superiores al 50 y $70 \%$ en agua caliente y lavado de ropa a caer a niveles por debajo del $8 \%$ en ambos dominios. Finalmente, en 'iluminación' aunque los cambios no fueron tan drásticos como en los otros servicios, el nivel en general se observó muy bajo (de $42 \%$ a $28 \%$ ).

\section{Discusión}

Los resultados presentados en la sección anterior sustentan el planteamiento que hicimos sobre los dos ejes del problema de investigación: i) los servicios de energía en los hogares presentan una desigualdad espacial-territorial en México a escala municipal; y, ii) el acceso a la energía es una condición necesaria pero insuficiente para mejorar la calidad de vida de la población, por lo cual debemos enfocarnos también en el acceso a los servicios que brinda la energía en los hogares.

Los resultados en la sección anterior demuestran que existe desigualdad espacial-territorial desde la óptica de los servicios de energía. Podría decirse que en algunos dominios específicos como el de 'alimentación' e 'iluminación' se reportan niveles "similares" de acceso en los diferentes municipios del país independientemente del nivel de electrificación (acceso a la electricidad). Sin embargo, en servicios de energía como higiene y limpieza, confort térmico (mayoritariamente por los materiales de construcción en viviendas), e 'información, comunicación, y entretenimiento' se observan un grado de polarización considerablemente alto.

Destacamos que los niveles de desigualdad que acabamos 


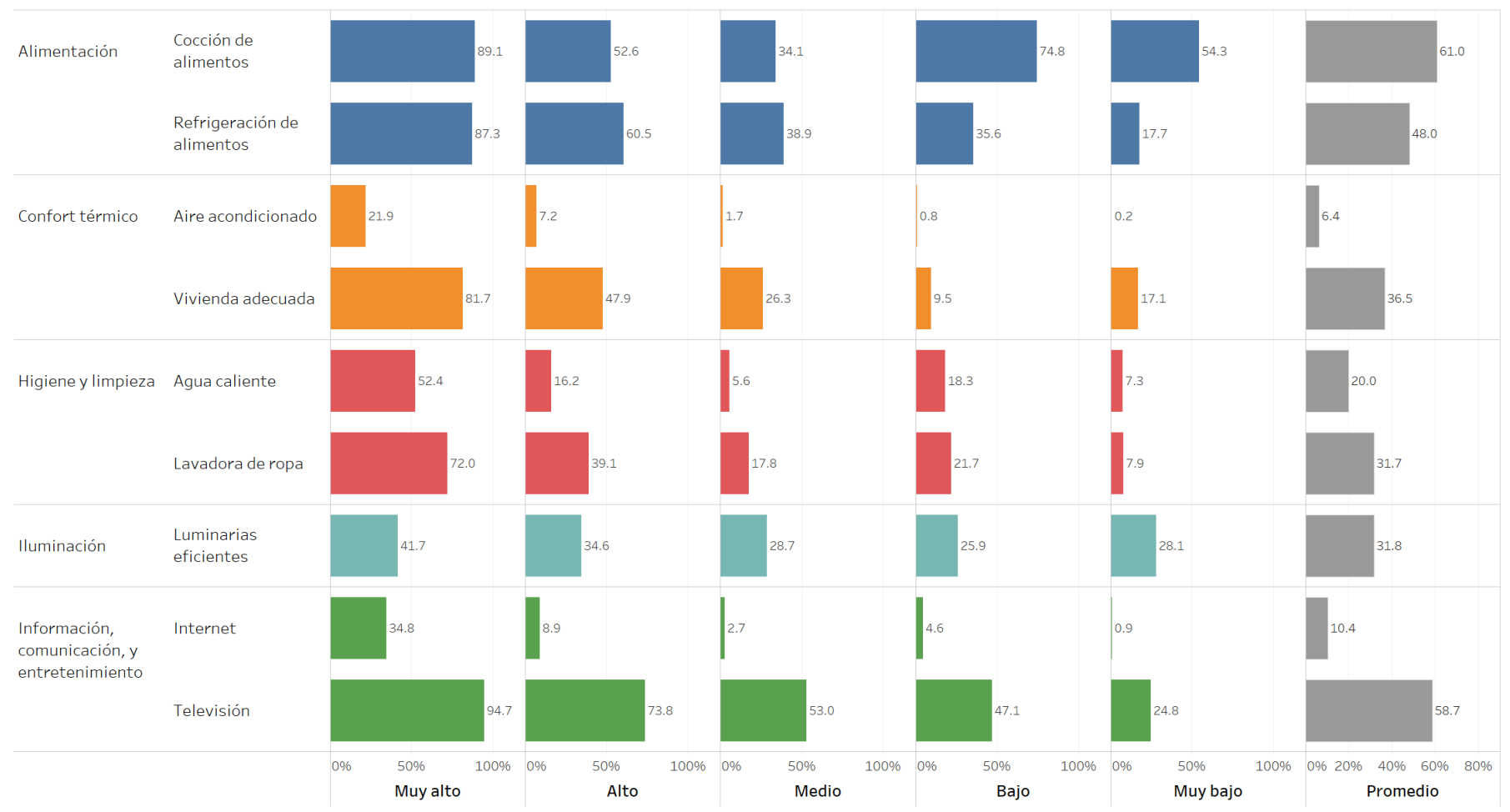

Figura 3: Hogares con acceso a los servicios de energía por categoría de acceso a electricidad. Fuente: elaboración propia / Figure 3: Households with energy access by electricity access level category

de describir se presentan en los cuatro grupos de municipios que clasificamos de acuerdo con sus niveles de acceso a la electricidad, aunque obviamente con distintos niveles de intensidad. Esto nos da pie a tocar el segundo eje, ya que mostramos que existe una clara desigualdad entre acceso a la energía y acceso a los servicios de energía. Es decir, aunque la mayoría de las viviendas de México están conectadas al servicio público de electricidad, una proporción significativa de estas viviendas tienen niveles de acceso bajos en los dominios de los servicios que esta brinda, entre los que sobresalen el uso de internet, el uso de luminarias eficientes, el uso de equipos de climatización, de calentadores de agua y de equipo de lavado de ropa (en este orden). Con estos resultados es posible identificar los servicios de energía que requieren mayor énfasis en futuros programas sociales que promuevan el bienestar humano y el desarrollo sustentable en México.

Ante estos nuevos hallazgos, hemos construido una serie de mapas del territorio nacional en función de los niveles de acceso a los principales servicios que brinda la energía en los hogares. Estos mapas se muestran en las figuras AG1 a AG10 del Apéndice Geográfico (Ver Material Suplementario). Consideramos que esta información es un insumo empírico con el cual se podrían realizar análisis a mayor profundidad sobre la relación entre energía y desarrollo. Adicionalmente, el lector puede utilizar el mapa interactivo, publicado en el sitio web de esta revista, el cual hemos construido con la información procesada en este trabajo.

\section{Conclusión}

El acceso a los servicios de energía en los hogares presenta una desigualdad espacial-territorial que sustenta la pertinencia del objetivo de este trabajo. El conocimiento generado en cada uno de los municipios de México es un insumo empírico para futuros estudios e investigaciones científicas y de política pública que aborden la relación entre energía, desarrollo y medio ambiente.

Por ejemplo, con la información generada se pueden realizar estudios que estimen el consumo de energía y emisiones de gases de efecto invernadero procedentes de los diferentes servicios de energía en cada municipio del país, con lo cual se podrían estimar escenarios de eficiencia energética y mitigación de emisiones en un período de tiempo determinado.

Otra línea de investigación que se puede desarrollar es la relación entre el uso de los diferentes servicios de energía con variables económicas y espaciales, por ejemplo, con los niveles de ingreso per cápita, pobreza, o ámbito urbano-rural de cada municipio.

Por otra parte, un tema que está cobrando cada vez más relevancia científica en México es el de pobreza energética y, la información que ofrece este trabajo a nivel municipal, representa un insumo para abordar dicho tema a escala subnacional.

Por último, México se ha comprometido en la Agenda 2030 de desarrollo sostenible a lograr el acceso universal a la electricidad, y una de las condiciones para lograrlo es saber precisamente donde se encuentran los hogares que aún no tienen 
acceso. En este trabajo desarrollamos una nueva visión del acceso la electricidad a través de los servicios que provee como una perspectiva más incluyente del problema que el objetivo siete de desarrollo sostenible trata de resolver.

\section{Agradecimientos}

Los autores agradecen los comentarios de dos revisores anónimos.

\section{Financiamiento}

Los autores agradecen el apoyo económico para este proyecto por el Consejo Nacional de Ciencia y Tecnología (Conacyt) No. CB-2015-258913-S, y el apoyo de KAKENHI No 19K14020 por la Sociedad Japonesa de la Promoción de la Ciencia (JSPS) y el apoyo del Instituto de las Américas por el proyecto Bala California Community-Based Needs Assesment and Stakeholder Mapping Focused on Energy Poverty and Inclusion.

\section{Referencias}

Cravioto, J., 2020. Los servicios de energía y su relación con la medición de pobreza energética en hogares. En: R. García Ochoa (Ed.), Pobreza energé- tica. Visiones del Sur. Colegio de la Frontera Norte (COLEF), Tijuana, BC, México.

Cravioto, J., Yamasue, E., Okumura, H., Ishihara, K. N., 2014. Energy service satisfaction in two Mexican communities: A study on demographic, household, equipment and energy related predictors. Energy Policy 73, 110 126, https://doi.org/10.1016/j.enpol.2014.04.031

Fell, M. J., 2017. Energy services: A conceptual review. Energy Research \& Social Science 27, 129-140, https://doi.org/10.1016/j.erss.2017.02.010.

García, E., 2004. Modificaciones al Sistema de Clasificación Climática de Köppen. Universidad Nacional Autónoma de México, México.

García Ochoa, R., Bracamonte Sierra, A., 2019. Acceso a los servicios de energía. Una crítica a la Agenda 2030 de México. Región y Sociedad 31, e1146e1146, https://doi.org/10.22198/rys2019/31/1146.

Hair, J. F., Anderson, R. E., Tatham, R. L., Black, W. C., 2007. Análisis multivariante. Prentice Hall, Madrid.

Instituto Nacional de estadística y Geografía (INEGI), 2016. Encuesta Intercensal 2015 [Set de datos]. INEGI, https://www.inegi.org.mx/programas/intercensal/2015/\#Microdatos.

Kalt, G., Wiedenhofer, D., Görg, C., Haberl, H., 2019. Conceptualizing energy services: A review of energy and well-being along the Energy Service Cascade. Energy Research \& Social Science 53, 47-58, https://doi.org/10.1016/j.erss.2019.02.026.

Naciones Unidas (NU), 2018. Hacia una nueva agenda de desarrollo sostenible [Página web]. Https://www.un.org/sustainabledevelopment/es/mdgs/.

This article accompanies the following material: Interactive map: 10.22201/igg.25940694e.2021.1.86.173 Suppl. Material: $\quad$ 10.22201/igg.25940694e.2021.1.86.175 